\title{
Índice de funcionalidade de portais eletrônicos municipais: o acesso à informação em
}

\section{saúde}

\author{
Index of functionality of municipal electronic portals: access to health information \\ Índice de funcionalidad de los portales electrónicos municipales: el acceso a la información sobre \\ salud
}

\author{
Suzely Adas Saliba Moimaz \\ ORCID: https://orcid.org/0000-0002-4949-529X \\ Universidade Estadual Paulista, Brasil \\ E-mail: suzely.moimaz@unesp.br \\ Carolina Carvalho Menez Pinto Nascimento \\ ORCID: https://orcid.org/0000-0003-0494-428X \\ Universidade Estadual Paulista, Brasil \\ E-mail: carolinacarv@hotmail.com \\ Tânia Adas Saliba \\ ORCID: https://orcid.org/0000-0003-1327-2913 \\ Universidade Estadual Paulista, Brasil \\ E-mail: tania.saliba@unesp.br \\ Ana Cláudia Festucci de Herval \\ ORCID: https://orcid.org/0000-0002-3367-3565 \\ Universidade Federal de Lavras, Brasil \\ E-mail: anafestucci@outlook.com \\ Fabiana Costa Machado Zacharias \\ ORCID: https://orcid.org/0000-0003-1150-6114 \\ Universidade de São Paulo, Brasil \\ E-mail: fabiana.zacharias@usp.br \\ Ricardo Alexandre Arcêncio \\ ORCID: https://orcid.org/0000-0003-4792-8714 \\ Universidade de São Paulo, Brasil \\ E-mail: ricardo@eerp.usp.br \\ Cléa Adas Saliba Garbin \\ ORCID: https://orcid.org/0000-0001-5069-8812 \\ Universidade Estadual Paulista, Brasil \\ E-mail: clea.saliba-garbin@unesp.br
}

\section{Resumo}

Este estudo buscou investigar em que medida as informações em saúde vêm sendo disponibilizadas à população e o grau de funcionalidade dos sites quanto ao acesso às páginas eletrônicas oficiais dos municípios sede dos Departamentos Regionais de Saúde (DRS) do Estado de São Paulo. Procurou, também, verificar quais características sociodemográficas, econômicas e de saúde contribuem para explicar a funcionalidade dos portais eletrônicos municipais. Trata-se de um estudo de caráter descritivo-exploratório, conduzido em 17 cidades do estado de São Paulo. Apurou-se o Índice de Funcionalidade (IF) através da criação de um instrumento com 34 itens divididos em 4 dimensões. Posteriormente, configurou-se uma Regressão Linear Múltipla com as variáveis explicativas população, PIB, IDH-M, cobertura da Atenção Primária à Saúde, da Estratégia Saúde da Família e da Saúde bucal para verificar possíveis associações com o IF. A média do IF foi 18,35; dp. 4,39; mediana 18; mínimo 11 e o máximo 29. O modelo estatístico indicou que não houve associação estatisticamente positiva entre o IF e as variáveis explicativas. A disponibilização das informações em saúde nos portais eletrônicos municipais apresentou baixo IF, em grande parte deles. Não houve relação positiva entre o IF das páginas e suas características sociodemográfica, econômicas e de saúde.

Palavras-chave: Acesso à informação; Participação da comunidade; Indicadores sociais; Serviços de saúde bucal; Gestão da informação em saúde.

\footnotetext{
Abstract

This study sought to investigate the extent to which health information has been made available to the population and the degree of functionality of the sites regarding access to the official websites of the municipalities that host the Regional Health Departments (DRS) of the State of São Paulo. To verify which sociodemographic, economic and health characteristics contribute to explain the functionality of municipal electronic portals. This is a descriptive-
} 
exploratory study, conducted in 17 cities in the state of São Paulo. The Functionality Index (IF) was determined by creating an instrument with 34 items divided into 4 dimensions. Subsequently, a Multiple Linear Regression was configured with the explanatory variables population, GDP, HDI-M, coverage of Primary Health Care, the Family Health Strategy and oral health to verify possible associations with the IF. The mean of the IF was 18.35; sd. 4.39; median 18; minimum 11 and maximum 29. The statistical model indicated that there was no statistically positive association between the IF and the explanatory variables. The availability of health information on municipal electronic portals showed low IF, in most of them. There was no positive relationship between the IF of the pages and their sociodemographic, economic and health characteristics.

Keywords: Access to information; Community participation; Social indicators; Dental health services; Health information management.

\section{Resumen}

Este estudio buscó investigar en que medida las informaciones en salud ha sido puesto a disposición de la población y el grado de funcionalidad de las webs en cuanto al acceso a las páginas electrónicas oficiales de los municipios que albergan los Departamentos Regionales de Salud (DRS) del Estado de São Paulo. También buscó verificar cuáles características sociodemográficas, económica y de salud contribuyen para explicar la funcionalidad de los portales electrónicos municipales. Se trata de un estudio descriptivo-exploratorio, conducido en 17 ciudades del estado de São Paulo. Se filtró el Î́ndice de Funcionalidad (IF) a través de la creación de un instrumento con 34 itens divididos en 4 dimensiones. Posteriormente, se configuró una una Regresión Lineal Múltiple con las variables explicativas población, PIB, IDH-M, cobertura de la Atención Primaria a la Salud, de la Estrategia Salud de la Familia y de la Salud Bucal para verificar posibles asociaciones con el IF. La media del IF fue 18,35; desviación estándar 4,39; mediana 18; mínimo 11 y el máximo 29. La plantilla estadística indicó que no hubo asociación estadísticamente positiva entre el IF y las variables explicativas. La disponibilidad de las informaciones en salud en los portales electrónicos municipales presentó bajo IF, en gran medida de ellos. No hubo relación positiva entre el IF de las páginas y sus características sociodemográfica, económicas y de salud.

Palabras clave: Acceso a la información; Participación de la comunidad; Indicadores sociales; Servicios de salud dental; Gestión de la información en salud.

\section{Introdução}

Nos últimos anos tem havido uma grande evolução tecnológica na disseminação das informações em todos os setores da atividade humana. A rapidez e qualidade na transmissão dos dados, proporcionadas pelas Tecnologias de Informação e Comunicação (TIC), configuraram um novo estágio do desenvolvimento civilizatório - a sociedade da informação.

A Internet pode ser considerada um mecanismo indutor de crescimento e desenvolvimento, e traz impactos socioeconômicos e no modo de vida das pessoas (Almeida, Spinola, Araújo, \& Reis, 2020). Como em qualquer outro setor, a gestão pública foi influenciada pelo avanço das TIC, o que possibilitou maior fluxo de informações entre governos e sociedade, ampliou a voz do cidadão e pode promover uma maior transparência por parte do Estado (Pinheiro et al., 2016; Moraes, 2014). Para uma governança transparente, é importante que haja a disponibilização das informações que favoreçam o empoderamento da coletividade nos processos decisórios do setor público (Valle-Cruz, 2019; Andrade, Raupp, \& Pinho, 2017).

Com a implantação do Sistema Único de Saúde (SUS) e a Lei orgânica da Saúde no 8080 de 19 de setembro de 1990 houve um intenso processo de descentralização que conferiu aos municípios a responsabilidade da gestão dos serviços, e o amplo acesso aos cuidados de saúde para grande parte da população brasileira, além da garantia da participação do cidadão neste setor, destacada na lei ${ }^{\circ} 8142$ de 28 de dezembro de 1990 (Paim, 2018).

Mais tarde, a Lei de Acesso à Informação (LAI) n. 12.527 (Brasil, 2011), fortaleceu a participação e o controle popular sobre a gestão pública. A LAI representou uma mudança de paradigma em relação à transparência pública (TP), pois o acesso à informação passa a ser a regra e o sigilo, a exceção, ou seja, qualquer indivíduo pode obter informações públicas, exceto aquelas classificadas como sigilosas (Brasil, 1988; Controladoria-Geral da União, 2011).

As informações divulgadas pela administração pública, em suas páginas da internet, podem auxiliar os cidadãos na resolução de suas demandas, principalmente na questão do acesso aos serviços, que é um dos principais estigmas do sistema de saúde (Nunes et al., 2016; Viacava, Oliveira, Carvalho, Laguardia, \& Bellido, 2018). Para isso, é preciso que os dados sejam 
inteligíveis e que alcancem o maior número possível de cidadãos. Eles devem proporcionar, a qualquer indivíduo, a assimilação e compreensão das informações disponíveis (Abdala \& Torres, 2016).

Apesar da participação popular estar assegurada na legislação brasileira, a consulta a dados públicos para conhecimento e posicionamento por parte da população e, principalmente, como etapa necessária para subsidiar as discussões e tomadas de decisões, ainda apresenta alguns entraves. Estudos realizados nos portais eletrônicos dos setores públicos, mostraram, com grande frequência, páginas de difícil acesso; desatualizadas; com informações, nem sempre, de fácil entendimento (Abdala \& Torres, 2016); e que priorizam somente o cumprimento da legislação (Andrade, Raupp, \& Pinho, 2017; Abdala \& Torres, 2016; Ribeiro, Ferreira, \& Teixeira, 2017).

Pesquisas buscaram avaliar a publicação dos dados nas páginas eletrônicas dos setores públicos com relação à questões financeiras, relatórios contábeis e fiscais (Vermeer \& Styles, 2019; Cruz, Ferreira, Silva, \& Macedo, 2012; Barros, Barros, Souza, Batista, \& Câmara, 2017; Filho, Colares, \& Andrade, 2015; Rossoni, \& Beiruth, 2016); informações gerais sobre o município, gestor municipal e vereadores (Cruz et al., 2012; Rossoni, \& Beiruth, 2016); navegabilidade dos portais (Cruz et al., 2012; Rossoni, \& Beiruth, 2016; Machado, Sonza, \& Aires, 2017); legislação municipal e instrumentos de planejamento (Cruz et al., 2012; Rossoni, \& Beiruth, 2016; Machado, Sonza, \& Aires, 2017); interação com os cidadãos e com a sociedade (Cruz et al., 2012; Rossoni, \& Beiruth, 2016); informações quantitativas e qualitativas sobre a gestão (Cruz et al., 2012; Barros et al., 2017; Rossoni, \& Beiruth, 2016); itens exigidos na LAI (Machado, Sonza, \& Aires, 2017; Comin, Ramos, Zucchi, Favretto, \& Fachi, 2016; Brocco, Grando, Martins, Brunozi Jr, \& Corrêa, 2018); que buscaram identificar as expectativas dos cidadãos em relação ao que é publicado nos portais eletrônicos do setor públicos (Valle-Cruz, 2019; Twizeyimana, \& Andersson, 2019). e que trouxeram uma maior abordagem política em detrimento a gerencial (Pinho, Sampaio, Winkler, \& Morais, 2019). Entretanto, estudos sobre o acesso e disponibilização das informações na área da saúde são escassos (Holeman, Cookson, \& Pagliari, 2016) e pesquisas que analisam a relação entre essa oferta e possíveis fatores explicativos não foram observados na literatura científica, nos últimos 10 anos, o que representa uma significativa lacuna no entendimento do assunto.

Embora tenha havido grandes avanços no acesso à informação através das TIC e das legislações referentes ao SUS e à transparência pública, muitos desafios ainda precisam ser superados. Neste sentido, o escopo nesta pesquisa foi investigar em que medida as informações em saúde vêm sendo disponibilizadas à população e o grau de funcionalidade dos sites quanto ao acesso às páginas eletrônicas oficiais dos municípios sede dos Departamentos Regionais de Saúde (DRS) do Estado de São Paulo. Este trabalho teve ainda, como objetivo, verificar a relação entre características; sociodemográficas, econômicas e de saúde e a funcionalidade dos portais municipais.

\section{Metodologia}

Trata-se de um estudo de caráter descritivo-exploratório, de natureza quantitativa (Pereira, Shitsuka, Parreira, \& Shitsuka, 2018), no qual se quantificou, por meio do uso de medições de grandeza, as informações disponíveis nos portais eletrônicos municipais. A pesquisa foi conduzida no estado de São Paulo, notadamente nos 17 DRS, os quais são responsáveis por coordenar as atividades da Secretaria Estadual de Saúde de São Paulo (SES/SP) no âmbito regional e promover a articulação intersetorial com os municípios e organismos da sociedade civil (Secretaria de Estado da Saúde de São Paulo, 2020).

Para o desenvolvimento da pesquisa foi adaptado um instrumento de coleta de dados e modelo de análise (Sales, 2012), também utilizado por em outro estudo no Brasil (Abdala \& Torres, 2016). Para adaptação do instrumento foram realizados estudos das legislações que abordam a questão da transparência pública, participação popular (Brasil 2011; 1998), e as que fazem referência à saúde bucal (Ministério da Saúde, 2004; 2006; 2018) e gestão no âmbito do SUS (Ministério da 
Saúde, 2005; 2016). O instrumento foi estruturado em quatro dimensões distintas: A - Acessibilidade/Usabilidade; B Prestação de informações básicas ao cidadão em SB; C - Controle social sobre a administração e D - Interatividade e participação.

A - Acessibilidade/usabilidade (7 pontos): Possibilidade de alteração do tamanho da fonte contraste; tradutor em libras; mapa do site; motor de busca; botão de "voltar"; logotipo da prefeitura em todas as páginas; e link para a Secretaria Municipal de Saúde (SMS) antes da "dobra";

B - Prestação de informações básicas ao cidadão em saúde bucal (SB) (10 pontos): Informações sobre os gestores (Secretário de Saúde e Coordenador de Saúde Bucal); Identificação e horários de atendimento das Unidades de Saúde com atendimento odontológico (na atenção básica ( $\mathrm{AB}$ ) e especialidades); escala odontológica; agendamento online; aplicativo para agendamento para dispositivo móvel; tempo de espera para encaminhamento para especialidades; acompanhamento online do encaminhamento para as especialidades; cobertura de procedimentos odontológicos ofertados pelo município; boletim epidemiológico da COVID-19 e informações sobre pactuação intermunicipal para a SB;

C - Controle social sobre a administração (12 pontos): Portal da Transparência; dados atualizados (últimos 90 dias); possibilidade de download dos documentos; Plano Municipal de Saúde (atual gestão); Programação Anual de Saúde (ano 2019); Relatório Anual de Gestão em Saúde (ano 2019); Prestação de Contas em Saúde (ano 2019); pactuação dos indicadores de saúde (SISPACTO - 2019); composição de recursos humanos em SB (Dentistas, Auxiliar em saúde bucal e Técnico em Saúde Bucal); atas das reuniões do Conselho Municipal de Saúde; Plano de Contingência para a COVID-19; e Protocolo para a COVID-19;

D - Interatividade e participação (5 pontos): "Fale Conosco" por e-mail; "Fale Conosco" por telefone"; perguntas frequentes; local específico para demandas dos usuários (denúncias, dúvidas e aquisição de informações); e redes sociais.

O instrumento adaptado foi elaborado com 34 itens, cujas respostas foram binárias (atributos identificados e não identificados) nos portais eletrônicos das prefeituras. Para a análise dos itens utilizou-se o Índice de Funcionalidade (IF) dos portais que foi representado pelo somatório de cada atributo em cada dimensão, conforme equação: $\mathrm{IF}=\sum A+\sum B+\sum C+\sum D$, sugerida pela literatura (Cruz et al., 2012).

Para verificação dos itens adaptados do instrumento, bem como suas pontuações e forma de análise, utilizou-se a técnica de consenso, denominada Comitê Tradicional (Souza, Silva, \& Hartz, 2015), envolvendo as pesquisadoras e especialistas em Saúde Bucal, permitindo uma discussão aberta e troca de ideias.

A dimensão "acessibilidade/usabilidade" refere-se ao primeiro contato do usuário com o site, e para ela foi analisado o desing/layout da página, através da deteçcão, do título, o corpo do texto, o menu, as hiperligações e o caminho de retorno à página inicial. Neste sentido, foram considerados os seguintes aspectos: se a informação era acessível a todos, inclusive àqueles portadores de deficiência e se o portal possuía elementos que facilitassem a usabilidade, como mapa e motor de busca; botão de "voltar"; presença de elementos de identidade visual, como logotipos que identificassem o sítio, não somente na página inicial, mas em todas as páginas acessadas; e se a informação mais importante ou o link para a mesma, estavam localizados antes da "dobra". A "dobra" é um termo jornalístico, definido como o ato de dobrar o jornal ao meio para facilitar a leitura. No caso da internet, a dobra é a primeira rolagem e em todas as páginas a parte do conteúdo mais importante deve estar acima desta. Para este estudo convencionou-se que o link para a SMS deveria estar antes da dobra (Ministério do Planejamento, Orçamento e Gestão, 2010; 2014; Nielsen, 2000).

Em relação à dimensão "Prestação de Informações Básicas ao Cidadão em SB", buscou-se identificar referências que pudessem satisfazer os cidadãos sobre os processos para obtenção de acesso aos serviços de saúde. Os itens avaliados nesta dimensão foram estabelecidos com base na literatura (Moimaz et al., 2017). Além dos dados mencionados anteriormente, foi identificada a presença ou ausência, do boletim epidemiológico da COVID-19. 
Na dimensão "Controle Social sobre a administração" foi observado se os sítios investigados cumpriam a LAI, pelo menos nos dados referentes à área da saúde, disponibilizando, além de receitas e despesas, resultados de programas, projetos, ações, bem como metas e indicadores propostos; atualização das informações e possibilidade de gravação das mesmas para facilitar, ao cidadão, a sua análise. Os itens selecionados para compor essa categoria foram baseados em publicações da Comissão Intergestores Tripartite; Departamento Nacional de Auditoria, ambas do Ministério da Saúde; e na LAI (Brasil, 2011; Ministério da Saúde, 2005; 2016).

A última categoria analisada "Interatividade e participação", focou na existência de ferramentas de relacionamento entre o cidadão e o município por meios eletrônicos, interações por redes sociais, locais para recebimento de informações demandadas pelos usuários e espaço para denúncias e dúvidas (Brasil, 2011).

A etapa de coleta de dados ocorreu de forma on-line, na busca dos endereços dos referidos portais. A procura foi efetuada nos meses de maio de 2020 a julho de 2020 e para realizar a procura das páginas dos municípios, utilizou-se a plataforma on-line do Google, admitindo-se como referência a locução "Prefeitura Municipal de [nome do município]". Após aceder ao portal foram investigadas a presença ou ausência dos dados, informações e serviços disponíveis.

Nesta pesquisa foi considerada como variável dependente o IF dos portais eletrônicos municipais, de natureza quantitativa, que variou de 0 a 34, resultado da aplicação do instrumento de coleta de dados nas páginas eletrônicas municipais. Como variáveis explicativas foram considerados os seguintes indicadores sociodemográficos e econômicos: população (POP) (número de habitantes em cada município) (Instituto Brasileiro de Geografia e Estatística, 2020a); produto interno bruto (PIB) per capita (Indicador do nível de atividade econômica do município (Instituto Brasileiro de Geografia e Estatística, 2020b); índice de desenvolvimento humano municipal (IDHM) (medida resumida do progresso a longo prazo em três dimensões básicas do desenvolvimento humano: renda, educação e saúde) (Programa das Nações Unidas para o Desenvolvimento, 2020).

Com relação às variáveis específicas do setor saúde, foram incluídas na pesquisa: cobertura da Atenção Primária à saúde (Cob/AP), tida como a "cobertura populacional estimada na Atenção Básica, dada pelo percentual da população coberta por equipes da Estratégia Saúde da Família e por equipes de Atenção Básica tradicional equivalentes e parametrizadas em relação à estimativa populacional"; cobertura da Estratégia Saúde da Família (Cob/ESF) sendo a "cobertura populacional estimada pelas equipes da Estratégia Saúde da Família, dada pelo percentual da população coberta por essas equipes em relação à estimativa populacional"; e a cobertura de Saúde Bucal na Atenção Primária (Cob/SB) referindo-se a "cobertura populacional estimada pela Saúde Bucal na Atenção Básica, dada pelo percentual da população coberta por equipes de Saúde Bucal vinculadas às Equipes de Saúde da Família e por equipes de Saúde Bucal equivalentes e parametrizadas na Atenção Básica tradicional em relação à estimativa populacional” (Ministério da Saúde, 2020).

Inicialmente, foi construída a Matriz de Correlação de Pearson, com o objetivo de aferir o grau de correlação entre duas variáveis. A aplicação desse teste foi realizada para verificar se variáveis explicativas, com o mesmo comportamento (colinearidade), afetariam os estimadores (beta) do modelo, o que não ocorreu.

Como o objetivo da análise foi modelar o IF em função das demais covariáveis, foi realizada uma Regressão Linear Múltipla. Assim, o IF de cada município $i(i=1, \ldots, 17)$ está relacionado com as variáveis explicativas, através do seguinte modelo: $I F_{i}=\beta_{0}+\beta_{1}(E S F)_{i}+\beta_{2}(S B)_{i}+\beta_{3}(A P)_{i}+\beta_{4}(P O P)_{i}+\beta_{5}(I D H)_{i}+\beta_{6}(P I B)_{i}+$ erro $_{i}$.

Neste modelo, IF é a variável dependente; as variáveis explicativas ou covariáveis são os indicadores socioeconômicas POP, PIB per capita e o IDHM e as coberturas, em porcentagem, da estratégia saúde da família (ESF), SB e atenção primária (AP); já descritas anteriormente. O parâmetro a ser estimado $\beta_{0}$ representa o valor do IF quando as 
covariáveis são nulas; os termos $\beta_{j}(\mathrm{j}=1, \ldots, 6)$ são os coeficientes da regressão; e o erro ${ }_{i}$ é o erro aleatório associado à i-ésima observação.

No modelo de regressão linear múltipla, optou-se por não utilizar intercepto, pois não havia interpretação prática para ele, uma vez que não é possível ter todas as variáveis explicativas nulas. Além disso, inicialmente, utilizaram-se as seis variáveis explicativas e, ao realizar o ajuste, apenas o parâmetro relacionado à variável IDHM foi significativo ao nível de 0,05 pelo teste $\mathrm{t}$.

Este modelo não satisfez às pressuposições de normalidade dos erros, então, foi executada uma transformação na variável resposta $Y^{\prime}=1 / I F$, através do método de Box Cox. Além disso, métodos de seleção de variáveis foram realizados, considerando todos os subconjuntos de covariáveis possíveis, visando obter o maior coeficiente de determinação ajustado (Adjusted $R$ Square $-R^{2}$ ). Como resposta desse processo de seleção de variáveis, obteve-se um modelo apenas com as variáveis Cob/SB e IDHM. As mesmas variáveis foram selecionadas utilizando o método sequencial Stepwise. Desta maneira, o modelo final ajustado é: $1 / I F_{i}=\beta_{1}(S B)_{i}+\beta_{2}(I D H)_{i}+$ erro $_{i}$, cujas estimativas obtidas foram 0,0015365 (erro padrão: 0,0001429) para $\beta_{1}$ e 0,0461262 (erro padrão: 0,0078791) para $\beta_{2}$, ambas significativas com valor-p $<0,01 \mathrm{e}$ estatística t iguais a 3,590 e 5,854, respectivamente. De acordo com a Resolução no 466/12 e a Resolução n. 510/16 da Comissão Nacional de Ética em Pesquisa, por se tratar de um estudo com dados secundários e de domínio público, não foi necessário submissão ao comitê de ética.

\section{Resultados}

A Figura 1 apresenta o comportamento das quatro dimensões que compuseram o IF. O estudo revelou, na análise comparativa do desempenho dos municípios nas quatro dimensões, que a maior pontuação ocorreu para a categoria D Interatividade e participação. Entretanto, somente este item não foi suficiente para que fosse atingido um alto nível de funcionalidade, o que refletiu no índice consolidado, uma vez que os outros três parâmetros apresentaram pontuações baixas. 
Figura 1. Gráficos dos totais de pontos por município para cada uma das quatro dimensões do instrumento de coleta. São Paulo, Brasil, 2020.
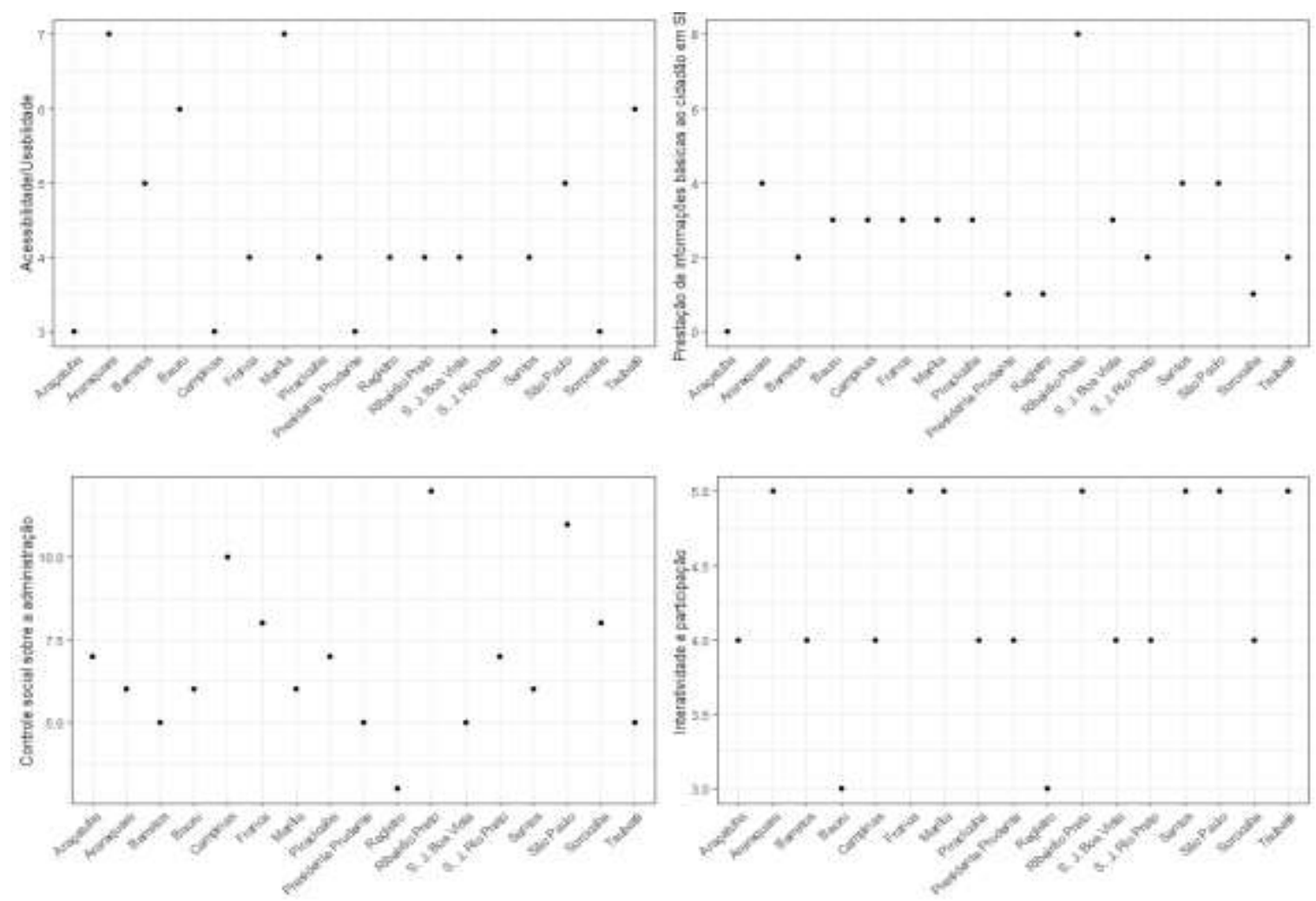

Fonte: Autores (2020).

Pode-se observar na Figura 1 que, para a dimensão A - Acessibilidade/Usabilidade, apenas Araraquara e Marília obtiveram pontuação máxima (7), seguidas por Bauru e Taubaté (6). Já para B - Prestação de Informações Básicas em SB, apenas Ribeirão Preto obteve pontuação máxima (10); as demais cidades obtiveram no máximo a metade destes pontos. O município de Ribeirão Preto também liderou a pontuação para o item C - Controle social sobre a administração, seguido por São Paulo. Para o último critério, D - Interatividade e participação, grande parte dos municípios fizeram 4 e 5 pontos. 
Figura 2. Gráficos do Índice de Funcionalidade, dispersão (à esquerda) e boxplot (à direita). São Paulo, Brasil, 2020

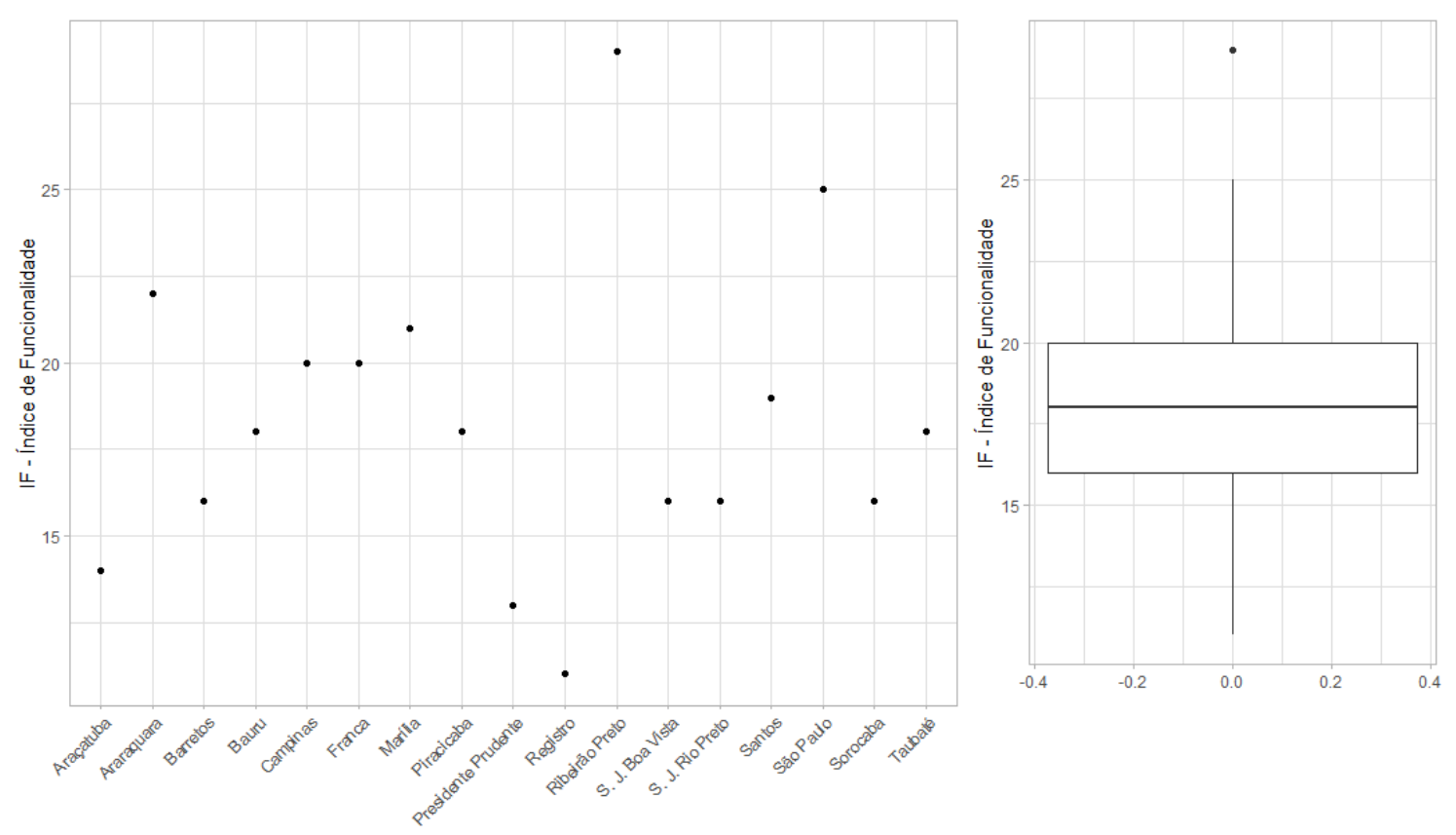

Fonte: Autores (2020).

Na Figura 2, no gráfico de dispersão, fica evidente o destaque de Ribeirão Preto, que se mostrou um outlier, com 29 pontos, uma vez que a variável de interesse, IF, é a soma das quatro dimensões anteriores. Ainda na Figura 2, pode-se observar que a média do IF dos municípios foi 18,35; $(d p=4,39)$, e o valor mínimo foi 11 . Além disso, no boxplot, percebe-se que metade dos municípios obtiveram pontuações inferiores à aproximadamente metade do total de pontos (mediana=18).

Para as variáveis explicativas, um resumo estatístico das mesmas, consta na Tabela 1 com as medidas de média, mediana, máximo, mínimo e desvio-padrão dos indicadores. Nela pode-se observar algumas assimetrias, principalmente com relação à população, às Coberturas de Saúde Bucal dos municípios e à Cobertura da Atenção Primária, frente as demais coberturas.

Tabela 1. Dados descritivos dos municípios sede das DRS do estado de São Paulo, segundo variáveis explicativas. São Paulo, Brasil, 2020

\begin{tabular}{cccccc}
\hline Variável & Média & Desvio Padrão & Mediana & Mínimo & Máximo \\
\hline Cob/AP* & 63,75 & 16,72 & 61,85 & 24,38 & 100,00 \\
Cob/ESF $^{\dagger}$ & 43,32 & 22,74 & 41,83 & 34,9 & 100,00 \\
Cob/SB $^{*}$ & 40,06 & 18,70 & 342125,00 & 54216,00 & 11869660,00 \\
População & 1046648,24 & 2802256,59 & 38402,18 & 28040,28 & 59788,23 \\
PIB $^{\S}$ & 43549,67 & 9927,42 & 0,80 & 0,75 & 0,84 \\
IDHM $^{\|}$ & 0,80 & 0,02 & & \\
\hline
\end{tabular}

${ }^{*} \mathrm{Cob} / \mathrm{AP}=$ cobertura da Atenção Primária à saúde; ${ }^{\dagger} \mathrm{Cob} / \mathrm{ESF}=$ cobertura da Estratégia Saúde da Família; ${ }^{\star}$ Cob/SB = cobertura de Saúde Bucal na Atenção Primária; ${ }^{\circledR}$ PIB = produto interno bruto; "IDHM = índice de desenvolvimento humano municipal. Fonte: Autores (2020). 
A Tabela 1 mostra que a Cobertura da AP é maior que a da ESF e que a de Saúde Bucal, variando de 24,38\% à 100\%, com desvio de 16,72. Pode ser observado, ainda, que para a variável População, existe uma discrepância entre o mínimo de 54.216 em Registro e o máximo de quase 12 milhões de habitantes, na cidade de São Paulo, sendo que, a segunda maior foi Campinas com pouco mais de 1 milhão de habitantes (Instituto Brasileiro de Geografia e Estatística, 2020a). Outra variável que também apresentou assimetria foi a Cobertura de Saúde Bucal com a máxima de 91,08\% no município de Registro, média de 40,06\% e desvio padrão de 18,70, o que mostra que as demais localidades não atingiram nem $60 \%$ de cobertura. Já para o IDH e o PIB não houve grandes disparidades.

A Figura 3 apresenta a Matriz de Correlação de Pearson demonstrando o grau de correlação, duas a duas, entre as variáveis, sendo que acima da diagonal, em azul, se encontram as correlações positivas e em vermelho, as correlações negativas, enquanto abaixo da diagonal, encontram-se os coeficientes de correlação de Pearson para todas as combinações de variáveis duas a duas.

Figura 3. Correlograma das variáveis dependente e explicativas dos municípios sede das DRS do estado de São Paulo. São Paulo, Brasil, 2020.

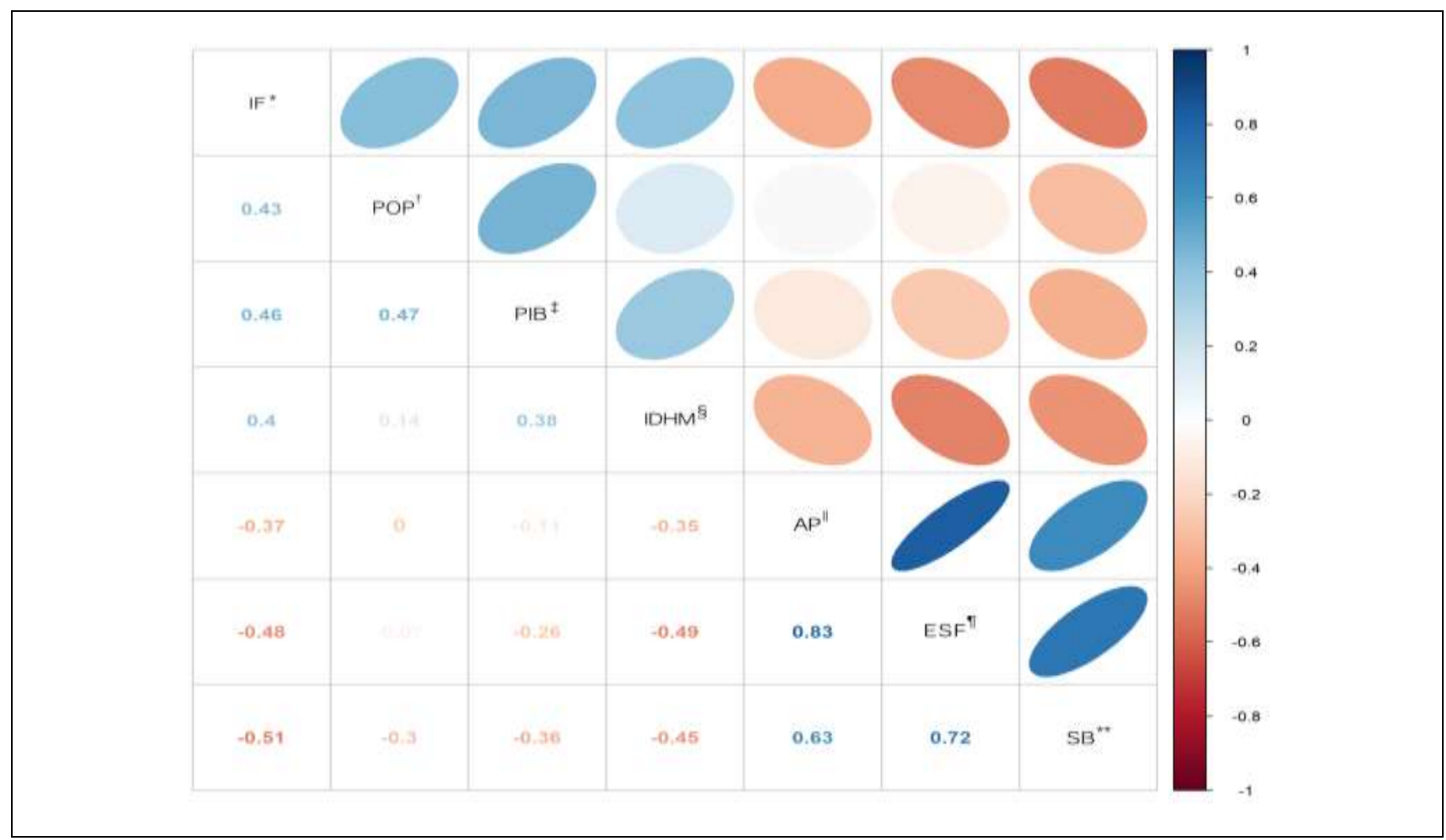

${ }^{*} \mathrm{IF}=$ índice de funcionalidade; ${ }^{\dagger} \mathrm{POP}=$ população; ${ }^{\dagger} \mathrm{PIB}=$ produto interno bruto; ${ }^{\S} \mathrm{IDHM}=$ índice de desenvolvimento humano municipal; ${ }^{\|} \mathrm{AP}=$ atenção primária; ${ }^{\mathbb{T}} \mathrm{ESF}=$ estratégia saúde da família; ${ }^{* *} \mathrm{SB}=$ saúde bucal. Fonte: Autores (2020).

No correlograma da Figura 3, adotando o nível de significância de 5\%, não foi observada correlação positiva fraca entre o IF e as três variáveis explicativas sociodemográficas e econômicas, POP $(\rho=0,43$, valor-p: 0,085$)$ PIB $(\rho=0,46$, valor-p: $0,06)$ e IDHM ( $\rho=0,40$, valor-p: 0,10$)$. Por outro lado, apesar de a correlação negativa fraca entre o IF e as coberturas Cob/AP ( $\rho=-0,37$, valor-p: 0,15$)$, Cob. ESF ( $\rho=-0,48$, valor-p: 0,052$)$ também não serem significativas ao nível de 5\%, para Cob/SB ( $\rho=-0,51$, valor-p: 0,04 ) houve uma correlação negativa moderada estatisticamente significativa. As três coberturas (AP, ESF e SB) apresentaram correlações positivas entre elas (Figura 3) mas que não afetaram os estimadores (beta) do modelo. 
O coeficiente de determinação ajustado (Adjusted $R$ Square $-R^{2}$ ), que mede o grau de ajustamento da reta de regressão às observações e indica a proporção da variabilidade da variável dependente que é explicada pelas variáveis independentes, para este modelo foi 0.9657. Isto quer dizer que 96,57\% da variabilidade da variável dependente (o inverso do IF) pode ser explicada pelas variáveis Cob/SB e IDHM. Os pressupostos da regressão linear foram atendidos no modelo proposto. O diagnóstico dos resíduos foi realizado via gráficos e através de testes.

É importante notar que, apesar dos valores dos coeficientes serem positivos, à medida que se tem maiores valores para Cob/SB e IDHM, são maiores, também, os valores de 1/IF, ou seja, menor o valor do IF.

A partir da análise de regressão, como foi ajustado o inverso do IF, a relação entre as variáveis explicativas e o índice não é linear. quando se mantém o IDHM fixo, igual ao seu valor médio (0.7975294), a cada 5 unidades de pontos em Cob/SB, o IF diminui a uma taxa que varia entre $0,39 \%$ para valores baixos de Cob.SB e $0,36 \%$ para valores mais altos de Cob/SB. Já quando se mantém a Cob/SB fixa, igual ao seu valor médio (40.06471), a cada 0,01 "unidades de variação" de IDHM, o IF diminui a uma taxa quase constante de aproximadamente $2,8 \times 10^{-5} \%$.

\section{Discussão}

Neste estudo sobre disponibilização de informação no setor saúde nas páginas de municípios do estado de SP, ficou evidente que o desempenho dos portais eletrônicos com relação ao IF ainda é incipiente, já que nenhum município atendeu a todos os 34 itens analisados. Ao se definir o IF dos portais eletrônicos das prefeituras, a dimensão "Prestação de Informações Básicas em SB" foi a que exibiu os piores resultados, onde somente um município atingiu mais de $50 \%$ dos itens avaliados. Essa dimensão, em particular, está relacionada à oferta de informações que auxiliam o cidadão no entendimento do acesso aos serviços de saúde. É preciso que haja dados disponíveis para o empoderamento do usuário com relação ao acesso, já que ele é um dos pilares da cobertura universal (Abiiro, \& Allegri, 2015).

Esse cenário de insuficiente publicação de informações é paradoxal, pois o Brasil possui uma das mais modernas legislações acerca do tema (Brasil, 2011; 1998) e investe em esforços para sua boa implementação em âmbito federal, mas as respostas reduzidas por parte dos municípios acabam por frustrar os resultados esperados (Andrade, Raupp, \& Pinho, 2017).

Distintos aspectos estão envolvidos na baixa funcionalidade dos portais eletrônicos municipais com relação ao acesso e disponibilização das informações. Um estudo (Moraes, 2014) destaca a questão da fragmentação das informações pela pulverização dos níveis administrativos, a dissipação de recursos e o paralelismo de ações. Esse contexto é acentuado pela progressiva deterioração das instâncias de gerenciamento da informação, que pode impor um problema à qualificação de recursos humanos, fragilizando as interlocuções e retardando inovações, esvaziando a potência e a função estratégica das TIC, que atendam às demandas da população. Também existem limites no que se refere aos investimentos nas instâncias públicas em informação, já que estes são feitos de forma pontual, isolada e programada de forma estanque nas esferas do governo (Moraes, 2014).

A questão da disponibilização das informações, através dos portais eletrônicos municipais, é importante não só pelo prisma da transparência, mas também pela função do Estado como gestor dessas informações e fundamental cooperador para a evolução da participação democrática da sociedade (Holeman, Cookson, \& Pagliari, 2016). Existe necessidade de os gestores públicos proporcionarem recursos para que os usuários tenham mais compreensão sobre as cidades onde as informações são divulgadas, em maior quantidade e qualidade, e que possam demandar por mais transparência junto às suas prefeituras (Rossoni, \& Beiruth, 2016).

É notória a necessidade de transformação da sociedade, com o objetivo de se manter informada sobre os atos do governo e seus direitos (Lodenstein, Dieleman, Gerretsen, \& Broerse, 2017), já que o acesso à informação é um fator que 
interfere nas relações sociais e contribui para viabilização da participação popular no setor saúde. São essas debilidades em promover a inclusão dos cidadãos nos processos decisórios, tão imprescindíveis no exercício da cidadania e no controle social, que tornam a administração pública, ainda, distante de uma gestão em saúde moderna, eficiente e equânime (Ribeiro, Ferreira, \& Teixeira, 2017). A implementação desse acesso configura-se numa conjuntura promissora de maior aproximação, da sociedade aos complexos problemas característicos do setor saúde (Moraes, 2014).

Nesta pesquisa, após a contextualização através da definição do IF, ao se utilizar a Matriz de Correlação de Pearson, era esperado que houvesse uma correlação positiva entre o IF dos sítios municipais e os indicadores sociodemográficos e econômicos (POP, PIB e IDHM), o que não ocorreu. Acreditava-se, da mesma forma, que maiores coberturas da AP, ESF e SB, correspondessem a melhores IF, entretanto, isso não se confirmou. O teste mostrou, ainda, uma forte correlação entre as três coberturas (AP, ESF e SB), entretanto o mesmo não aconteceu entre estas e os indicadores sociodemográficos e econômicos (POP, PIB e IDHM).

Testando associações entre um indicador de desenvolvimento social municipal e indicadores de serviços públicos odontológicos em municípios do sul do Brasil, um estudo (Godoi, Singh, Mello, Brennan, \& Peres, 2019) mostrou que um maior IDH foi associado a menor cobertura de serviço público de saúde bucal. Segundo estes autores isso pode ser explicado pela limitação de precisão dos indicadores de saúde bucal, pois estes atendem principalmente a objetivos de monitoramento de serviços e são incapazes de refletir as desigualdades subjacentes por fatores socioeconômicos e demográficos.

Ao se buscar identificar a relação entre indicadores sociodemográfico, econômicos e de saúde e a divulgação das informações em saúde, através do IF dos portais eletrônicos municipais, acreditava-se na hipótese, que seria possível estabelecer uma relação positiva entre a disponibilização das informações e as variáveis explicativas população, PIB per capita e IDHM, baseado em estudos realizados (Filho, Colares, \& Andrade, 2015; Rossoni, \& Beiruth, 2016; Machado, Sonza, \& Aires, 2017; Comin et al., 2016; Brocco et al., 2018). Entretanto os resultados do Modelo de Regressão Linear Múltipla não corroboraram com esta hipótese, talvez pelo fato de que o coeficiente de determinação ajustado (Adjusted $R$ Square $-R^{2}$ ), para esse estudo tenha sido de aproximadamente $97 \%$ e valores mais elevados de $\mathrm{R}^{2}$ conferem maior adequabilidade do modelo. Algumas análises similares utilizaram coeficientes menores como 55,7\% (Cruz et al., 2012); 57,9\% (Rossoni, \& Beiruth, 2016) e 35,06\% (Machado, Sonza, \& Aires, 2017).

Não houve relação positiva entre as variáveis coberturas da AP, ESF e SB, com o IF. Uma explicação para esse fato poderia estar relacionada à composição da amostra, com maioria de cidades de pequeno e médio porte. Um estudo (Uchôa et al., 2016) expôs a existência uma relação entre indicadores socioeconômicos, e o acesso aos serviços de saúde na AP, mostrando uma vulnerabilidade nos municípios menores. Nestas localidades, a escassez de recursos humanos e/ou tecnológicos poderiam justificar a ausência de dados e a demora na atualização das informações em saúde, o que seria capaz de causar dificuldades aos usuários que buscam, nas páginas eletrônicas municipais, orientações de como acessar os serviços de saúde nos territórios. É plausível que municípios menores apresentem condições insuficientes para manter atualizados os equipamentos necessários para disponibilização digital das informações e que enfrentem maiores dificuldades de compor seus quadros de funcionários, em número e com qualificações desejadas para o exercício das funções.

Pressupunha-se ainda, que cidadãos com melhores condições de saúde, educação e níveis satisfatórios de empregabilidade, teriam mais possibilidades de desempenhar o controle social, demandando dos gestores públicos a continuidade dessas condições. Um governo com melhor desempenho nessas áreas, da mesma forma, buscaria ratificar sua boa administração, publicitando os seus atos (Zuccolotto \& Teixeira, 2019). Todavia, há escassez de trabalhos relacionados ao grau de acesso e disponibilização de informações em saúde e SB, em portais eletrônicos públicos, e indicadores de saúde, o que acaba por obscurecer uma tentativa de explicação para esses fatos. 
Deve-se pontuar também a possibilidade de que exista, por parte dos gestores, falta de cultura de disponibilização de informações para planejamento e avaliação dos serviços e receio na divulgação de dados, devido à questão do uso da informação para efeitos político-partidários. Estudos revelam que as páginas eletrônicas públicas apresentam baixa interação do cidadão com o governo (Abdala \& Torres, 2016; Andrade, Raupp, \& Pinho, 2017; Michener, Contreras, \& Niskier, 2019); com número reduzido de dados disponibilizados (Cruz et al., 2012); e com informações "escolhidas a dedo" ou extensamente mediada por gestores públicos (Michener \& Bersch, 2013).

Pode-se pensar que, ainda que tenha havido avanços com as TIC, o SUS e a Política Nacional de Saúde Bucal (Ministério da Saúde, 2004), extensos vácuos e deficiências organizativas ainda estão presentes, especialmente em grandes centros urbanos, onde se condensam os problemas de integração dos variados serviços, devido à concentração histórica de diferenças institucionais e complexas demandas populacionais. Neste sentido, torna-se necessário repensar as estratégias para o aperfeiçoamento da integralidade da atenção. Este fato, demanda da gestão pública a inclusão do setor odontológico na assistência à saúde, através da implementação e construção de uma odontologia pública resolutiva, de maior qualidade, e que responda às expectativas e anseios da população.

Ao se tentar encontrar uma explicação para a associação negativa entre a Cob/SB e o IF, pensou-se que, municípios que possuem menores coberturas da AP, ESF e SB poderiam apresentar maiores facilidades em disponibilizar as informações, já que o número de informações que necessitariam ser ofertadas seria menor. Uma rede mais restrita demandaria por menos logística de TIC, contudo são hipóteses ainda a serem estudadas. A realização de pesquisas que envolvam a percepção dos gestores, trabalhadores da saúde e representantes da população nos conselhos de saúde possa, talvez, esclarecer pontos ainda obscuros e aprofundar questões relacionadas ao acesso à informação do setor público de saúde, por meio das páginas eletrônicas.

Os achados encontrados e as hipóteses levantadas, demonstram o tamanho do desafio para explicar a correlação entre os indicadores sociodemográficos, econômicos e de saúde com o acesso, disponibilização e transparência das informações em saúde nas páginas eletrônicas oficiais dos municípios.

Este estudo tem como limitação, o fato de ter avaliado somente a presença ou ausência dos itens de cada dimensão. A questão da qualidade e completude das informações e a real predisposição dos governos municipais em abrir um canal de interação com o usuário, não foram abordados. Isto exigiria a construção de um outro arcabouço metodológico.

\section{Considerações Finais}

A disponibilização das informações em saúde nos portais eletrônicos de municípios do estado de São Paulo é deficitária, com baixo IF em grande parte deles. Não houve relação positiva entre o IF das páginas das localidades estudadas e suas características sociodemográfica, econômicas e de saúde.

Esta pesquisa possibilitou identificar deficiências na disponibilização e acesso das informações em saúde, contribuindo para o reconhecimento dos pontos que necessitam de maior atenção por parte dos gestores públicos com vistas à melhora no atendimento à legislação sobre transparência pública e consequentemente ao empoderamento dos usuários dos serviços de saúde, no exercício do controle social.

Para estudos futuros, poderão ser realizadas análises mais aprofundadas, abrangendo as peculiaridades das informações disponibilizadas, num maior número e diversidades de municípios, utilizando também outras variáveis, a fim de reconhecer demais características e indicadores explicativos para o acesso à informação em saúde e em SB. 


\section{Referências}

Abdala, P. R. Z., \& Torres, C. M. S. O. (2016). A transparência como espetáculo: uma análise dos portais de transparência de estados brasileiros. Revista Administração Pública e Gestão Social, 8(3):147-58.

Abiiro, G. A., \& Allegri, M. (2015). Universal health coverage from multiple perspectives: A synthesis of conceptual literature and global debates. BMC International Health and Human Rights, 15(17):1-7.

Almeida, P. N. L., Spinola, C. A., Araújo, P. S. R., \& Reis, R. B. (2020). Acessibilidade tecnológica e novas fronteiras em Boipeba, Estado da Bahia, Brasil. Research, Society and Development, 9(11):e269119261.

Andrade, R. G., Raupp, F. M., \& Pinho, J. A. G. (2017). Em busca da transparência ativa em câmaras: uma investigação nos maiores municípios brasileiros. ASAA Journal, 10(1):3-20.

Barros, C. C., Barros, F. M. C., Souza, F. J. V., Batista, E. M., \& Câmara, R. P. B. (2017). Transparência na gestão pública: um estudo do atendimento dos municípios do estado Rio Grande do Norte a lei complementar n ${ }^{\circ}$ 131/2009. Revista Ambiente Contábil, 9(2):200-21.

Brasil. (1988). Constituição da República Federativa do Brasil de 1988. Brasília.

Brasil. (2011). Lei n. 12.527, de 18 de novembro de 2011. Regula o acesso a informações previsto no inciso XXXIII do art. $5^{\circ}$, no inciso II do $\S 3^{\circ}$ do art. 37 e no $\S 2^{\circ}$ do art. 216 da Constituição Federal; altera a Lei ${ }^{\circ} 8.112$, de 11 de dezembro de 1990; revoga a Lei ${ }^{\circ} 11.111$, de 5 de maio de 2005 , e dispositivos da Lei n ${ }^{\circ} 8.159$, de 8 de janeiro de 1991; e dá outras providências. Brasília: Diário Oficial da União.

Brocco, C., Grando, T., Martins, V. Q., Brunozi Jr, A. C., \& Corrêa, S. (2018). Transparência da gestão pública municipal: fatores explicativos do nível de transparência dos municípios de médio e grande porte do Rio Grande do Sul. Revista Ambiente Contábil, 10(1):139-59.

Comin, D., Ramos, F. M., Zucchi, C., Favretto, J., \& Fachi, C. C. P. (2016). A transparência ativa nos municípios de Santa Catarina: Avaliação do índice de atendimento à lei de acesso à informação e suas determinantes. Revista Catarinense da Ciência Contábil, 15(46):24-34.

Controladoria-Geral da União (BR). (2011). Acesso à Informação Pública: Uma introdução à Lei 12.527, de 18 de novembro de 2011. Brasília.

Cruz, C. F., Ferreira, A. C. S., Silva, L. M., \& Macedo, M. Á. S. (2012). Transparência da gestão pública municipal: Um estudo a partir dos portais eletrônicos dos maiores municípios brasileiros. Revista de Administração Pública, 46(1):153-76.

Filho, G. A. L., Colares, A. F. V., \& Andrade, I. C. F. (2015). Transparência da Gestão Fiscal Pública: um Estudo a Partir dos Portais Eletrônicos dos Maiores Municípios do Estado de Minas Gerais. Contabilidade Vista \& Revista, 26(2):114-36.

Godoi, H., Singh, A., Mello, A. L. S. F., Brennan, D.S., \& Peres, M. A. (2019). Area-level social development and indicators of public dental services in Southern Brazil. Community Dentistry and Oral Epidemiology, 47(3):274-80.

Holeman, I., Cookson, T. P., \& Pagliari, C. (2016). Digital technology for health sector governance in low and middle income countries: A scoping review. Journal of Global Health, 6(2):020408.

Instituto Brasileiro de Geografia e Estatística. (2020a). Cidades - Ribeirão Preto. Rio de Janeiro.

Instituto Brasileiro de Geografia e Estatística. (2020b). Produto Interno Bruto (PIB). Rio de Janeiro.

Lodenstein, E., Dieleman, M., Gerretsen, B., \& Broerse, J. E. W. (2017). Health provider responsiveness to social accountability initiatives in low- and middle-income countries: A realist review. Health Policy and Planning, 32(1):125-40.

Machado, V. N., Sonza, I. B., \& Aires, F. L. (2017). Fatores explicativos do nível de evidenciação dos maiores municípios do Brasil. Revista de Gestão Estratégica de Organizações, 5(3):31-47.

Michener, G., \& Bersch, K. (2013). Identifying transparency. Information Polity, 18(3):233-242.

Michener, G., Contreras, E., \& Niskier, I. (2019). Da opacidade à transparência? Avaliando a Lei de Acesso à Informação no Brasil cinco anos depois. Revista de Administração Pública, 52(4):610-29.

Ministério da Saúde. (2004). Diretrizes da política nacional de saúde bucal. Brasília.

Ministério da Saúde. (2005). Orientações técnicas sobre auditoria em odontologia no SUS. (2a ed.), Brasília.

Ministério da Saúde. (2018). Secretaria de Atenção à Saúde Departamento de Atenção Básica. A saúde bucal no sistema único de saúde. Brasília.

Ministério da Saúde. (2020). E-Gestor Atenção Básica: Informação e Gestão da Atenção Básica. Brasília.

Ministério da Saúde. (2006). Portaria $N^{o}$ 399, de 22 de fevereiro de 2006. Divulga o Pacto pela Saúde 2006 - Consolidação do SUS e aprova as Diretrizes Operacionais do Referido Pacto. Brasília.

Ministério da Saúde. (2016). Curso básico de regulação do Sistema Único de Saúde: SUS. Brasília.

Ministério do Planejamento, Orçamento e Gestão. (2010). Padrões Web em Governo Eletrônico e-PWG - Cartilha de Usabilidade. Brasília.

Ministério do Planejamento, Orçamento e Gestão. (2014). e-MAG versão 3.1: português (Brasil). Brasília. 
Moimaz, S. A. S., Burili, M. C., Bordin, D., Garbin, C. A. S., Saliba, T. A., \& Saliba, N. A. (2017). Satisfação dos usuários segundo variáveis de organização dos serviços públicos odontológicos. Archives of Health Investigation, 6(1):14-19.

Moraes, I. H. S. (2014). Governança e modelo de gestão da informação e inovação em sistemas e serviços de atenção à saúde. In: Cunha, F.J.A.P., Pereira, H.B.B., \& Lázaro, C.P., organizadores. Conhecimento, inovação e comunicação em serviços de saúde. Rio de Janeiro: Fiocruz. p. 19-41.

Nielsen, J. (2000). Projetando websites. Gulf Professional Publishing.

Nunes, B. P., Flores, T. R., Garcia, L. P., Chiavegatto Filho, A. D. P, Thumé, E., \& Facchini, L. A. (2016). Time trend of lack of access to health services in Brazil, 1998-2013. Epidemiologia e Serviços de Saúde, 25(4):777-87.

Paim, J.S. (2018). Thirty years of the Unified Health System (SUS). Ciência \& Saúde Coletiva, 23(6):1723-8.

Pereira, A. S., Shitsuka, D. M., Parreira, F.J., \& Shitsuka, R. (2018). Metodologia da pesquisa científica. UFSM.

Pinheiro, A. L. S., Andrade, K. T. S., Silva, D. O., Zacharias, F. C. M., Gomide, M. F. S., \& Pinto, I. C. (2016). Health management: the use of information systems and knowledge sharing for the decision making process. Texto Contexto-Enfermagem, 25(3):e3440015.

Pinho, J. A. G., Sampaio, R. C., Winkler, I., \& Morais, K. (2019). Digital democracy in the administration field: mapping the construction of the field in Brazil. Cadernos Gestão Pública e Cidadania, 24(78): e-73630.

Programa das Nações Unidas para o Desenvolvimento (BR). (2020). Desenvolvimento Humano e IDH. Brasília.

Ribeiro, M. C., Ferreira, V. A., \& Teixeira, R. A. (2017). Transparência na gestão em saúde: um estudo sobre a democratização do acesso à informação no estado de Minas Gerais. Revista Vozes dos Vales, 6(11):1-24.

Rossoni, F. V., \& Beiruth, A. X. (2016). Transparência na gestão pública municipal: análise nos sites das prefeituras do estado do Espírito Santo. Revista de Auditoria, Governança e Contabilidade, 4(17):1-16.

Sales, T. S. (2012). Acesso à informação, controle social das finanças públicas e democracia: análise dos portais da transparência dos estados brasileiros antes e após o advento da lei no 12.527/2011. Revista Direito Público, 9(48):28-48.

Secretaria de Estado da Saúde de São Paulo. (2020). DRS XIII - Ribeirão Preto. Ribeirão.

Souza, L. E .P. F., Silva, L. M. V., \& Hartz, Z. M. A. (2015). Conferência de consenso sobre a imagem-objetivo da descentralização da atenção à saúde no Brasil. In: Hartz, Z.M.A., Silva, \& L.M.V. Avaliação em saúde: dos modelos teóricos à prática na avaliação de programas e sistemas de saúde. Rio de Janeiro: Fiocruz. p. 65-102.

Twizeyimana, J .D., \& Andersson, A. (2019). The public value of E-Government - A literature review. Governament Information Quarterly, 36(2):167-78.

Uchôa, S. A. C., Arcêncio, R. A., Fronteira, I., Coêlho, A. A., Martiniano, C. S., Brandão, I. C. A, \& Maroto, R. M. (2016). Potential access to primary health care: What does the National Program for Access and Quality Improvement data show? Revista Latino-Americana de Enfermagem 2016;24:e2672.

Valle-Cruz, D. (2019). Public value of e-government services through emerging technologies. International Journal of Public Sector Management, 32(5):53045 .

Vermeer, B. Y., \& Styles, A. K. (2019). Online availability and accessibility of local government financial statements: is the public interest being served? Accounting and the Public Interest, 19(1):57-82.

Viacava, F., Oliveira, R. A. D., Carvalho, C. C., Laguardia, J., \& Bellido, J. G. (2018). SUS: supply, access to and use of health services over the last 30 years. Ciência \& Saúde Coletiva, 23(6):1751-62.

Zuccolotto, R., \& Teixeira, M. A. C. (2019). Transparência: Aspectos conceituais e avanços no contexto brasileiro. Brasília: Escola Nacional de Administração Pública. 